\title{
A Rare Case of Giant Neuroendocrine Tumor of the Thymus Treated with EN Bloc Resection
}

\author{
João Isidro Fracasso ${ }^{1,2}$, Antônio Benincá Albuquerque ${ }^{3}$, André Luca Boeira Rovani ${ }^{3}$, \\ Larissa Maroni $^{3}$, Guilherme Baroni de Macedo ${ }^{1,2}$, Leonardo Priori ${ }^{1,2}$, Vinícius Buaes Dal'Maso ${ }^{2,3}$, Tiago Teixeira \\ Simon, Pedro Moacir Braghirolli Braghini ${ }^{4}$, Cássia Beltrame ${ }^{2,3}$
}

${ }^{1}$ Departamento de Cirurgia, Hospital São Vicente de Paulo (HSVP). Passo Fundo, RS, Brasil.

${ }^{2}$ Clínica do Pulmão. Passo Fundo, RS, Brasil.

${ }^{3}$ Faculdade de Medicina, Universidade de Passo Fundo (UPF). Passo Fundo, RS, Brazil.

${ }^{3}$ Faculdade de Medicina, Universidade de Passo Fundo, Passo Fundo, RS, Brazil

${ }^{4}$ Departamento de Oncologia, Hospital São Vicente de Paulo (HSVP). Passo Fundo, RS, Brazil.

*Corresponding author - João Isidro Fracasso, Email - joaoisidrof@ hotmail.com

Received 2018-12-17;

Accepted 26 January 2019;

Published 03 February 2019

\begin{abstract}
Neuroendocrine tumors (NETs) are epithelial neoplasms with predominant neuroendocrine differentiation that arise in most organs of the body. Neuroendocrine tumors of the thymus (tNET) and mediastinum are very uncommon. We report the case of a 49-year-old male patient with neuroendocrine tumor of the thymus, superiorly in the anterior mediastinum, asymptomatic of the respiratory tract presenting with epigastric pain, treated with the lesion's surgical resection en bloc associated with radiotherapy and chemotherapy.
\end{abstract}

Keywords: Thymus; Neuroendocrine tumors of the thymus (tNET); Carcinoid tumor; Surgical resection

\section{Introduction}

Neuroendocrine tumors (NETs), defined as epithelial neoplasms with predominant neuroendocrine differentiation, arise in most organs of the body. Some of the clinical and pathologic features of these tumors are characteristic of the organ of origin, but other attributes are shared by neuroendocrine neoplasms (NENs) irrespective of their anatomic site ${ }^{[1]}$. Primary mediastinal NETs and neuroendocrine tumors of the thymus (tNET) are very rare neoplasms ${ }^{[2,3]}$. Clinically, the mediastinal tumors are often symptomless, being usually asymptomatic. When present, they commonly manifest local symptoms due to the compression chest pain or invasion of mediastinal structures (cough, dyspnoea, and eventual superior vena cava syndrome). Furthermore, systemic symptoms secondary to the tumor capacity to produce hormones or cytokines can be found (flushing, diarrhea, and wheezing ${ }^{[4-6]}$. Chest computed tomography scan is important to define the characteristics of the tumor and its anatomical relationships with surrounding structures. Definitive diagnosis is based on histopathological examination and immunophenotypic markers ${ }^{[7]}$;

\section{Case Report}

A 49-year-old male patient with history of epigastric pain was referred by the gastroenterologist to the pulmonologist due to chest $\mathrm{x}$-ray abnormalities during the evaluation of the patient's clinical feature. According to the chest $\mathrm{x}$-ray abnormalities, the patient was hospitalized to diagnostic investigation. Physical examination was normal. A chest computed tomography (CT) scan identified a heterogeneous, lobulated and expansive lesion with density of soft tissues located superiorly in the anterior mediastinum, measuring approximately $16.6 \times 12.2 \times 9.4 \mathrm{~cm}$, in the transverse, longitudinal and anteroposterior diameters, respectively (Figure 1). The lesion insinuates between the supra-aortic vessels, displacing the superior vena cava to the right; areas of contrast enhancement within the mass were observed, as well as hypoattenuating areas (presumed necrosis) and small coarse focus of calcification in its central portion. 


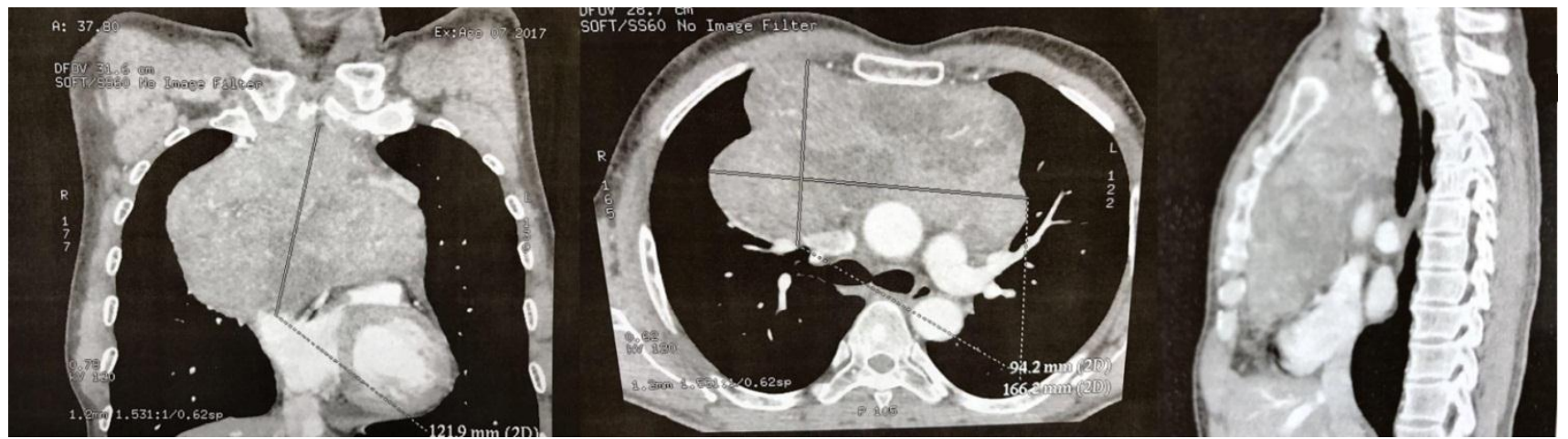

Figure 1: Computed Tomography of thorax evidencing a large expansive lesion measuring approximately $16.6 \times 12.2 \times 9.4 \mathrm{~cm}$ in the anterior mediastinum.

The patient was submitted to mediastinotomy with biopsy of the mass for etiological investigation. Pathology examination revealed a high-grade neuroendocrine neoplasia (grade 3) of the thymus, with extensive angiolymphatic invasion. The patient did not present symptoms of Carcinoid Syndrome. The patient was tested for the preoperative staging by bone scintigraphy, abdominal and cranial CT scans, all negative for metastasis. The treatment of choice was the non-curative surgical resection of the mass. The patient underwent a median sternotomy with the en bloc resection of the tumor mass (Figures 2 and 3), associated with partial pericardiectomy of all the anterior pericardium with resection of the pericardial fat tissue, having as posterior limit bilaterally the phrenic nerve. During the resection, partial invasion of the lesion into the superior vena cava was verified, being necessary the lesion resection and rrhaphy of the vena cava. Finally, the skin closure was done.

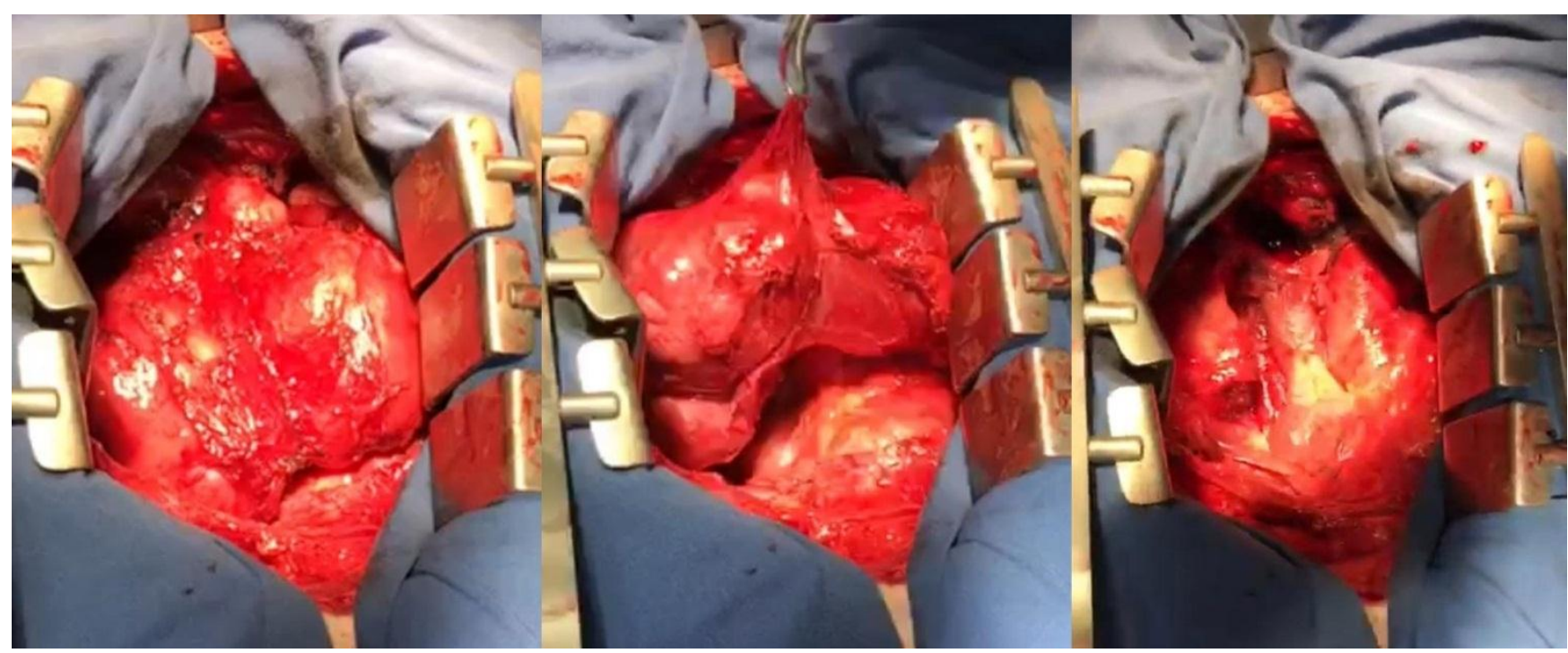

Figure 2: Resection of the lesion en bloc.

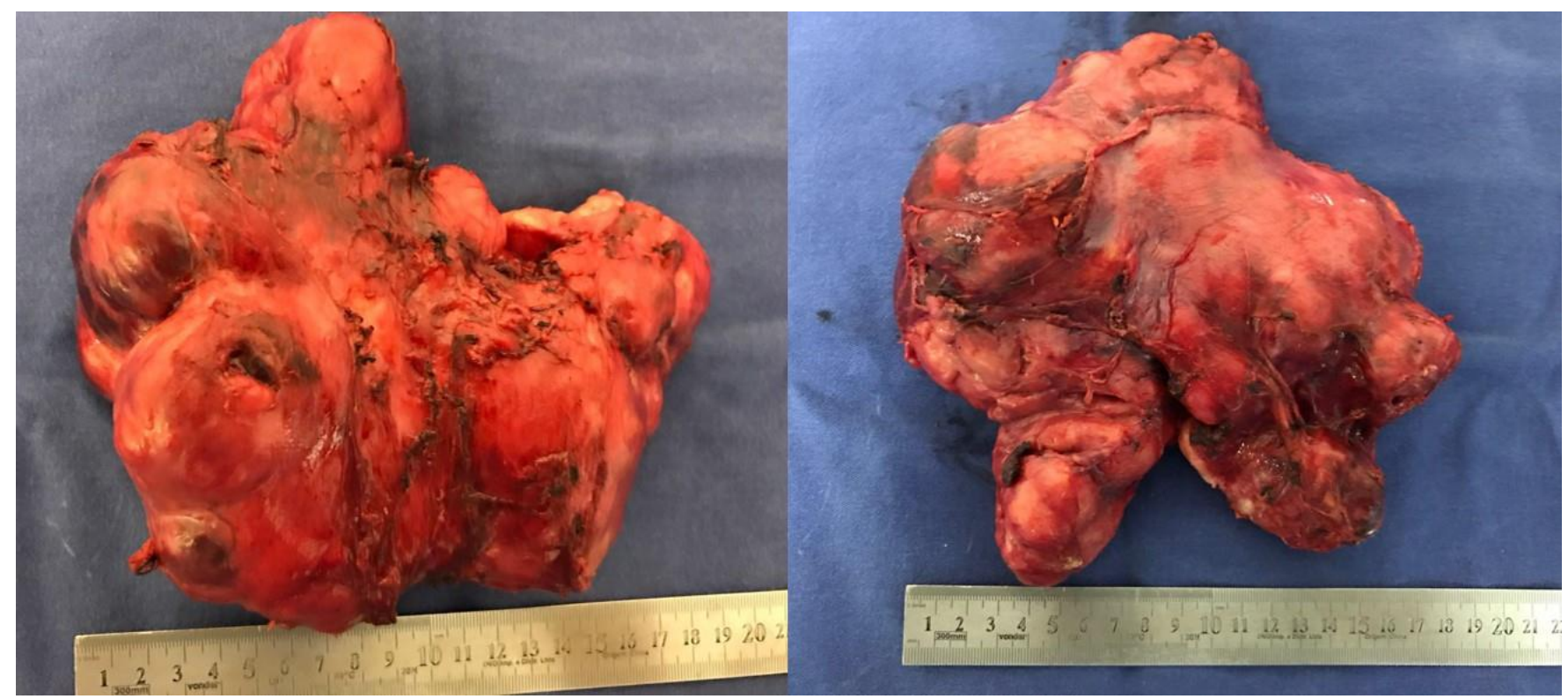

Figure 3: Resected tumor mass. 
The post-operative treatment was done with sessions of chemotherapy and radiotherapy, during five months, once the surgery was not curative. In this period, as consequence of radiotherapy the patient presented radiation pneumonitis. After the end of the sessions and improvement of the clinical features, the control chest CT showed: increase of mediastinal reticular opacities of both upper lobes (may be related to post-radiation alterations); can be noted also the appearance of centrolobular micro-nodules adjacent to the reticular opacities previous described, notably to the right; absence of signs of mediastinal lymph nodes enlargement; remain unaltered the atelectatic opacities in both lungs, notably in the left lower lobe; no signs of pleural effusion. Nowadays, the patient remains under medical follow-up.

\section{Discussion}

The carcinoids are a neuroendocrine group of tumors, arising from Kulchitsky cells. Carcinoid was defined in 1907 by Oberndorfer but is now more relevantly named as the neuroendocrine tumor ${ }^{[6]}$. Neuroendocrine neoplasms are a relatively rare (account for about $0.5 \%$ of all newly diagnosed malignancies) and heterogeneous tumor type, comprising approximately $2 \%$ of all malignancies, with a prevalence of less than 200,000 in the United States ${ }^{[8]}$. For functional purposes, NENs are divided into two groups on the basis of clinical behavior, histology, and proliferation rate: well differentiated (low grade to intermediate grade) neuroendocrine tumors and poorly differentiated (high grade) neuroendocrine carcinoma; this histological categorization/dichotomization is highly clinically relevant with respect to impact on treatment and prognosis even though it is not absolute since a subset of tumors with a low-grade appearance behaves similarly to high-grade lesions ${ }^{[8,9]}$. NETs may arise in any organs and tissues ${ }^{[7]}$. The most frequent primary sites are the gastrointestinal tract (62\%-67\%) and the lung $(22 \%-27 \%)^{[8]}$. NETs of the mediastinum are very rare; among these, tNET are the most frequent, they are exclusively located in the anterosuperior mediastinum and account for $2 \%$ to $4 \%$ of all mediastinal tumors ${ }^{[7]}$. Mediastinal tumors are often assymptomatic and and are occasionally detected by chest radiograph. When present, symptoms are caused by local tumor growth, distant metastases and/or endocrine manifestations. Local symptoms are the commonest, such as compressive chest pain, cough, dyspnoea, respiratory distress and superior vena cava syndrome $^{[3,5,10]}$. Functional NETs may rarely secret hormones, resulting in carcinoid syndrome, a clinical condition with symptoms such as skin flushing, diarrhea and palpitations ${ }^{[11,12]}$. These hormone-related symptoms are often an indicator of liver metastatic disease $\mathrm{e}^{[11]}$. Some NETs are non functional ${ }^{[2]}$. Paraneoplastic syndromes are rare in high grade tumors ${ }^{[3]}$. Computed tomography of thorax is used on diagnosis approach, as an important exam to know disease extent, involvement of various structures, vascular invasion and metastasis ${ }^{[2]}$. NETs have tendency for lymph node and distant metastasis, which are seen in about $50 \%$ of the patients. Bones and lungs are the main distant metastasis sites ${ }^{[3]}$. Confirmatory diagnosis requires a combination of anatomopathological and immunohistochemical examination ${ }^{[13]}$. Treatment of choice is surgical excision of the lesion, since absence of macroscopically radical primary resection and no surgical resection are negative prognostic factors in patients with
tNET, as well as high proliferative rate, measured by Ki67 index. In some cases quimio and/or radiotherapy may be used as adjuvant therapy, as well as in inoperable patients ${ }^{[10,13,14]}$. A study from Crona et al. suggests that Temozolomide or Platinum based chemotherapy should be considered as first-line medical therapy in patients with metastatic or non-resectable tumours ${ }^{[14]}$.

\section{References}

[1] Klimstra DS, Modlin IR, Coppola D, et al. The pathologic classification of neuroendocrine tumors: a review of nomenclature, grading, and staging systems. Pancreas 2010; 39:707-12.

[2] Dwivedi AN, Goel K, Tripathi S, et al. Primary neuroendocrine mediastinal tumor presenting with carcinoid syndrome and left supraclavicular lymphadenopathy: Clinicoradiological and pathological features. J Cancer Res Ther 2013;9, 278-80.

[3] Bohnenberger $\mathrm{H}$, Dinter $\mathrm{H}$, König $\mathrm{A}$, et al. Neuroendocrine tumors of the thymus and mediastinum. J Thorac Dis 2017;9(Suppl 15): S1448-S1457.

[4] Duwe BV, Sterman DH, Musani AI. Tumors of the mediastinum. Chest 2005; 128:2893-909.

[5] Macchiarini P, Ostertag H. Uncommon primary mediastinal tumours. The Lancet Oncology 2004; 5(2), 107-118.

[6] Aghajanzadeh M, Jafanegad A, Alive A, et al. Repot of Huge Primary Mediastinal Carcinoid Tumors with Horsiness. Int J Case Rep Short Rev 2017;3 (2): 016018.

[7] Ventura L, Gnetti L, Silini EM, et al. Primary atypical carcinoid tumor of the mediastinum: a very rare finding. J Thorac Dis 2017; 9:E367-72.

[8] Oronsky B, PC Ma, Morgensztern D, et al. Nothing But NET: A Review of Neuroendocrine Tumors and Carcinomas. Neoplasia 2017; 19 (12):991-1002.

[9] Bosman FT, Carneiro F, Hruban RH, Theise ND. 4th ed. WHO Press; Geneva, Switzerland: 2010. World Health, Organization (WHO) Classification of Tumours of the Digestive System.

[10] Brcic L, Heidinger M, Popper H. Neuroendocrine neoplasms of the mediastinum. Pathologe 2016; 37(5):434-40.

[11] Schmidt C, Bloomston M, Shah MH. Well-differentiated neuroendocrine tumors: a review covering basic principles to loco-regional and targeted therapies. Oncogene 2011; 30(13): 1497-1505.

[12] Soga J, Yakuwa Y, Osaka M. Evaluation of 342 cases of mediastinal/thymic carcinoids collected from literature: a comparative study between typical carcinoids and atypical varieties. Ann Thorac Cardiovasc Surg 1999; 5:285-92.

[13] Wang HL, Sun ZG, Xiao W, et al. Mediastinum primary small cell neuroendocrine carcinoma. Contemp Oncol (Pozn) 2016;20(1): 86-90.

[14] Crona J, Björklund P, Welin S, et al. Treatment, prognostic markers and survival in thymic neuroendocrine tumours. a study from a single tertiary referral centre. Lung Cancer 2013; 79:289-93. 